\title{
The Role of Neuroimaging in Developmental Social Psychology
}

\author{
Norihiro Sadato $\cdot$ Tomoyo Morita $\cdot$ Shoji Itakura
}

Received: 9 April 2008 / Accepted: 16 September 2008 /Published online: 10 October 2008

(C) The Author(s) 2008. This article is published with open access at Springerlink.com

\begin{abstract}
The development of social cognition is defined as the behavioral patterns, feelings, attitudes, and concepts that children manifest in relation to other people, and the way in which these different functions change with age. To understand the development of social cognition, modelling based on longitudinal behavioral observation is essential. Neuroimaging techniques will aid in this process by providing the neural basis of the psychological constructs, and the constraints for the model. Here, the issue of selfrecognition and self-evaluation is presented as an example. Technical advances will allow the application of functional neuroimaging techniques directly to babies and/or children, particularly under the age of 6 years old, in the near future.
\end{abstract}

Keywords Developmental psychology · Functional MRI . Near-infrared spectroscopy · NIRS · Self-recognition

\section{Developmental social psychology and neuroimaging techniques}

How do we become social beings? This developmental social psychology question is attracting growing interest in Japan in the face of recent reports on the declining birthrate,

\footnotetext{
N. Sadato $(\triangle)$

Department of Cerebral Research,

National Institute for Physiological Sciences,

Okazaki, Aichi, 444-8585, Japan

e-mail: sadato@nips.ac.jp

N. Sadato $\cdot$ T. Morita $\cdot$ S. Itakura

Japan Science and Technology Agency (JST) / Research Institute

of Science and Technology for Society (RISTEX),

Tokyo, Japan

S. Itakura

Department of Psychology, Kyoto University,

Kyoto, Japan
}

the breakdown in classroom discipline, and social withdrawal. Social cognition is defined as the processing of information that culminates in the accurate perception of the dispositions and intentions of other individuals (Brothers 1990). The development of social cognition is defined as the behavioral patterns, feelings, attitudes, and concepts that children manifest in relation to other people, and the way in which these different functions change with age (Schaffer 1996).

To understand the development of social cognition, initially we have to identify the elementary constructs of social cognition that can be measured and quantified. As with other psychological models, the models of developmental social cognition use hypothetical representations and the processes on them. There is no direct evidence supporting the existence of processes and representation: their existence must be inferred on the basis of the success of the model. However, hypothetical representation is sufficiently powerful to produce a limitless number of models to account for the data. Thus, some constraints are necessary (Willingham and Dunn 2003). Classically, reaction time or error/success rate in developmental psychology was the method used to provide such constraints. However, recent advances in neuroimaging techniques have provided another approach. Neuroimaging techniques such as functional MRI (fMRI) allow associations between the psychological constructs and specific human brain areas to be made non-invasively. The essence of this method is brain localization. Thus neuroimaging techniques are an additional way of differentiating between the models by their specific predictions for how fMRI response to the task would be affected by task parameters (Willingham and Dunn 2003).

Longitudinal behavioral data allow us to describe changes in behavior across age, that is, the chronological order of the emergence of functions $\mathrm{X}$ and $\mathrm{Y}$ (behavioral milestones). As the behavior should be tightly linked to the brain function, changes in behavior across age are conceiv- 
ably accompanied by the changes in the brain development which is reflected in the task-related activation pattern to the particular behavioral tasks. As the adult brain is the result of the development through the past, it is conceivable that adult fMRI can pinpoint the neural substrates of functions $\mathrm{X}$ and Y. Then their functional relationship could be further tested by other interventional techniques such as TMS (transcranial magnetic stimulation), or lesion studies in adults.

Identification of the neural correlates of behavioral milestones in adults is essential to collecting/analyzing child fMRI data when available. Furthermore, anatomical information provides important implications for the construction of the developmental model, because the information of the location-specific function is accumulated in the neuroscience field. Finally, neuroimaging studies could provide the way to test the hypothesis derived from the models.

An example is the mirror neuron system (MNS). MNS is first described by non-human primate experiments, followed by numerous functional neuroimaging studies (Iacoboni and Dapretto 2006, for review). The MNS is composed of inferior frontal gyrus, parietal lobe, and superior temporal sulcus. MNS is supposed to encode both goal-directed action and observation, and thus is closely related to imitation (Iacoboni and Dapretto 2006). Imitation is closely linked to the development of the theory of mind (Baron-Cohen et al. 1993). With the hypothesis that the core deficit of autism is the reduced ability of imitation that is mediated by MNS, an fMRI was conducted on children (around 12 years old) with autism spectrum disorder during an imitation of emotional facial expression (Dapretto et al. 2006). They found reduced task related activity in MNS of the autism group compared with control group, suggesting that a dysfunction of MNS underlies the social deficits of autism (Dapretto et al. 2006).

At present, applying the neuroimaging approach to pediatric populations is difficult due to technical limitations (Aslin and Mehler 2005), particularly under 6 years old. We therefore propose the following strategy for developmental social psychology. The elementary constructs of social cognition detected by behavioral analysis along the developmental time axis are localized in the adult brain. This information is used to provide constraints for the developmental model, which in turn is tested by longitudinal observation (Fig. 1). Here we present an example of this approach for studying the development of self-recognition and self-awareness (Morita et al. 2008) that occur in the ages of 1.5 to 3 year old.

\section{Self-awareness and self-recognition in adults}

As adults, we can experience extreme distaste and even aversion when we are shown our own images as recorded in a photograph or video. We can also become highly selfconscious when we unexpectedly see our own reflected image as we walk through the streets of a city. This reaction is caused by the self-awareness provoked by the feedback of our own image. There are two types of self-awareness: "public self-awareness" that is provoked when one accesses recognizable external information on the self such as one's face or body, and "private self-awareness" that is provoked when one accesses internal personal information that cannot be directly observed such as one's beliefs and thoughts. As

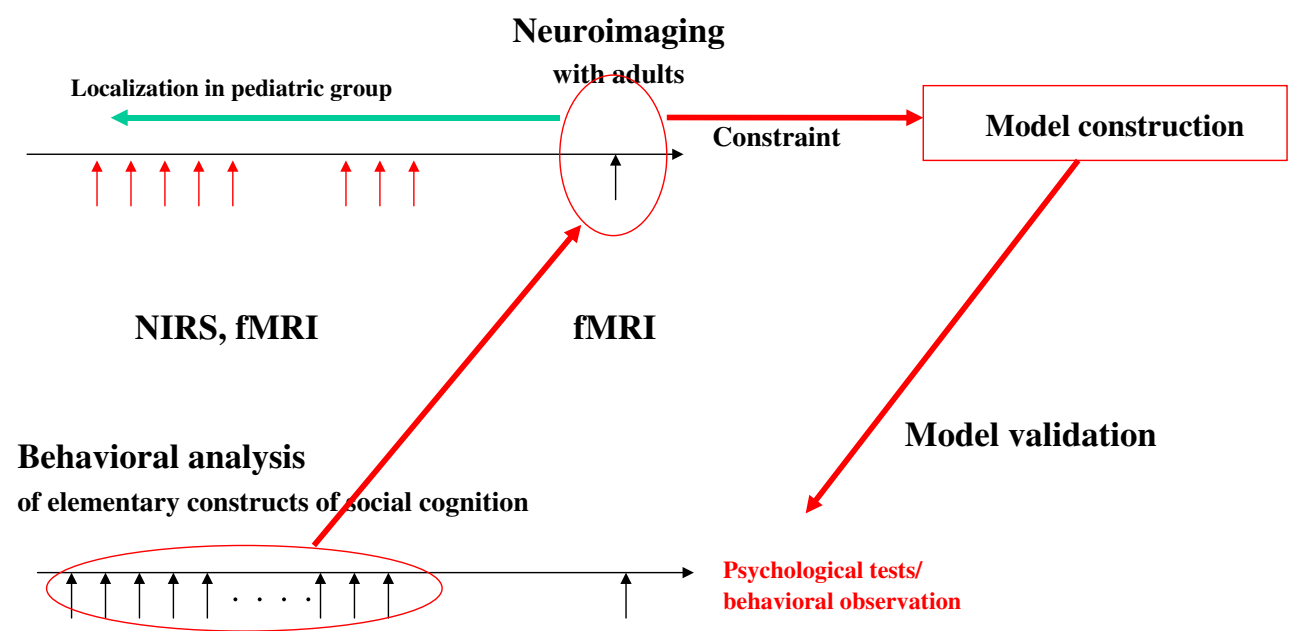

Fig. 1 An approach to social cognitive development with neuroimaging techniques. Horizontal black lines indicate the age of observation by means of neuroimaging (upper horizontal line) and behavioral analysis (lower horizontal line). Vertical arrows indicate the timing of the observation. Using adult fMRI, the neural substrates of the elementary constructs of social cognition detected by behavioral analysis are depicted. This information is used to provide constraints for the developmental model construction, which in turn is tested by longitudinal quantitative behavioral observation. Furthermore, identification of the neural correlates of the behavioral milestones in adults is essential to conduct/ analyze child neuroimaging data (red vertical arrows) when available 
private self-awareness is accompanied by a conceptual or abstract sense of self-awareness, it is considered to be a form of self-awareness at a higher level than that of public self-awareness (Morin 2006).

By directing attention towards the self, the perceptual feedback of self images initiates an automatic comparison against standards, which comprise mental representations of ideal behaviors or attitudes (Duval and Wicklund 1972). This mental process is defined as self-evaluation. If the actual self does not reach the standards that are set by the subject, he or she experiences negative feelings such as embarrassment and guilt. By contrast, if these standards are met, the subject experiences positive feelings such as pride. These feelings are classified as self-conscious emotions.

\section{Development of self-recognition and self-awareness}

When do children start recognizing themselves? The mark test devised by Gallup (1970) is widely used in the measurement of self-recognition in human infants and primates (Gallup 1970). In this method, subjects view themselves in a mirror after rouge has been secretly applied to their face. Rougedirected responses are used to infer self-recognition. Human infants aged between 6 and 11 months are frequently observed to react by laughing or touching the mirror, but they almost never touch their own bodies. This indicates that infants regard their own image in the mirror as that of a different person. However, once a child reaches the age of between 18 months and 2 years, he or she will touch and attempt to remove the lipstick or mark on the basis of the image reflected in the mirror (Amsterdam 1972). It is because these children realize that the reflected image is their own that they are able to touch their body and not the mirror. Such a child is thus thought to have acquired the ability of self-recognition. This test is used as an indicator of the emergence of the concept of self in the field of developmental psychology, and is also regarded as an important indicator of cognitive ability in the field of evolutionary psychology.

Soon after children are capable of recognizing their own image in a mirror, they demonstrate self-conscious or coy reactions upon seeing their own reflection in this way (Lewis et al. 1989). This is called exposure embarrassment, which is a feeling that accompanies an awareness of self, and is referred to as a "self-conscious emotion" in order to distinguish it from innate emotions such as joy and anger (Sullivan et al. 2003) (Fig. 2). At this developmental stage, the exposure embarrassment and other self-conscious emotions, such as empathy and jealousy, occasionally arise when a child becomes the center of attention or is the subject of praise (Lewis et al. 1989). Lewis and colleagues (Lewis 1997) conducted mark tests on infants aged 18 months during an experiment in which they were praised. They found that the emergence of self-recognition was linked to the exposure embarrassment produced by praise from other people. Bischof-Köhler (1991) indicates that self-recognition is linked to the sense of empathy (Bischof-Köhler 1991). This reaction is regarded as the manifestation of lower-level public self-awareness.

\section{Development of self-evaluation and high-level self-evaluation}

Whereas exposure embarrassment is evident in children at around 2 years of age, the sense of shame, evaluative embarrassment, guilt, and pride that emerge as a consequence of self-evaluation begin to appear when a child is around the age of 3 years. This corresponds precisely to the age when the process of self-evaluation emerges (Alessandri and Lewis 1993). The self-conscious emotion caused by selfevaluation is closely related to the acquisition of norms and rules with which one's behavior is compared (Lewis 1997). If inappropriate behavior that departs from the established criteria is discovered, this will be fed back in the form of negative feelings such as shame and embarrassment, and future actions will be revised accordingly. In other words, the self-conscious emotion evoked by self-evaluation might act as a modifier of one's own behavior and attitudes.

In summary, these developmental psychological findings imply that the neural representations of self-recognition and self-awareness might be distinct, and that different levels of self-awareness exist.

\section{Adult fMRI study of self-face recognition and self-evaluation}

To dissect the neural substrates of self-awareness at high and low levels, and those of self-face recognition (Platek et al.
Fig. 2 Development of self and self-conscious emotion (modified from Sullivan et al 2003)
Development of Self

\begin{tabular}{|l|l|l|l|}
\hline Age (Mo) & Construct & Related construct & Related self-conscious emotion \\
\hline 18 & Self recognition & & \\
\hline 24 & Self awareness low level & & $\begin{array}{l}\text { Exposure-embarrassment } \\
\text { empathy jealousy }\end{array}$ \\
\hline $30-36$ & Self awareness high level & Self evaluation & $\begin{array}{l}\text { Evaluative-embarrassment } \\
\text { guilty pride }\end{array}$ \\
\hline
\end{tabular}


2004; Sugiura et al. 2005, 2006; Uddin et al. 2005), we conducted an fMRI study with 19 adult normal volunteers (Morita et al. 2008). The first experimental hypothesis was that the brain regions that responded to evaluative embarrassment would reflect the process itself or the self-evaluation process that characterizes higher level self-awareness. This is because the feeling of evaluative embarrassment is caused by differences between immediate perceptions and standards. The second hypothesis was that the neural substrates of lowerlevel self-awareness (that is, public self-awareness) would be strongly recruited when participants who had a strong disposition to attend to the observable aspects of the self were exposed to feedback face images (public self-consciousness). This disposition can be measured by a questionnaire using the "self-consciousness scale", which assesses the personal trait of self-consciousness. This scale includes two subscales: a public self-consciousness subscale and a private self-consciousness subscale. The public self-consciousness subscale assesses the tendency to care about visible aspects of the self. The private self-consciousness subscale assesses the tendency to think about inner or abstract aspects of the self. Using these scores, we investigated the relationship between the personal trait of self-consciousness and the brain regions that are related to the evaluation of one's own facial appearance.

In order to induce embarrassment in the participants during the experiment, we prepared facial images selected from video-recordings. When the video-recordings were paused, it was possible to isolate unnatural or strange faces that deviated from the participants' normal photographic images, for example due to a widely open mouth or partly closed eyes (Fig. 3). Showing the participants unusual facial images of themselves was intended to cause them embarrassment. We prepared 21 images of each participant's own face and those of others. The participant's own facial images and those of others appeared in the center of a screen for $3 \mathrm{~s}$ in random order. During the session, the participants were required to rate how photogenic each face was by giving a score ranging from $1=$ "very bad" to $7=$ "very good". They were also required to press the button assigned to each score when a fixation cross appeared. Each session included 21 trials for the SELF condition, 21 trials for the OTHERS condition, and seven "null events" in which no stimulus was shown. We repeated each session four times.

Figure 3 shows the relationship between the photogenic score measured inside the scanner and the embarrassment score measured outside the scanner. Participants experienced strong embarrassment when they viewed their own face images evaluated as very bad. Thus, it was probable that the participants experienced embarrassment when they viewed their own faces during the fMRI experiment.

The embarrassment scores measured outside the scanner were used as parametric covariates in the analysis. This
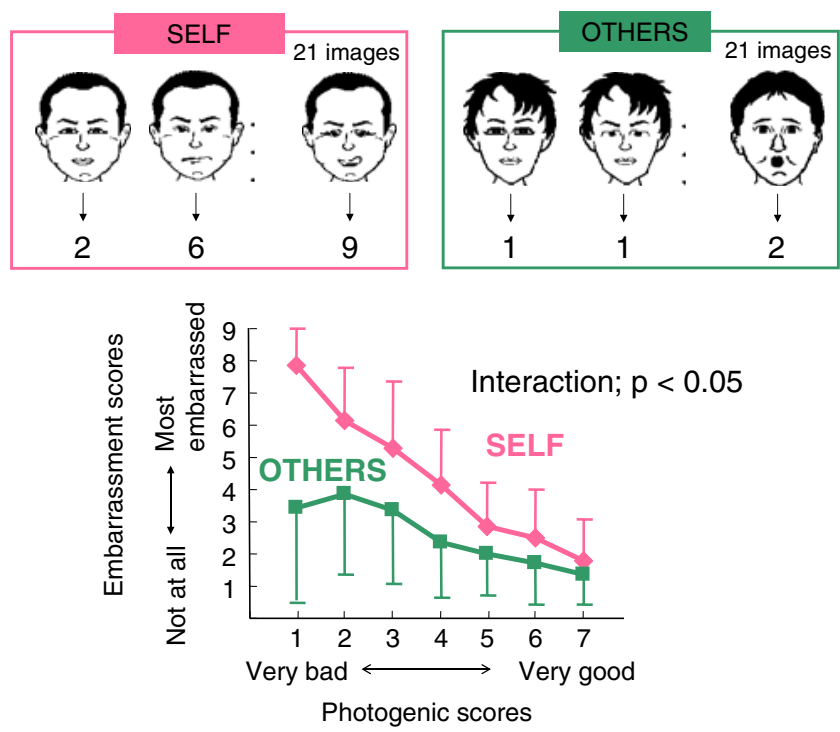

Fig. 3 (Top) Embarrassment scores. After MRI scanning, the participants rated how embarrassed they felt when they viewed their own or others' images by giving a score ranging from 1 to 9 . The larger the score, the greater the embarrassment. (Bottom) Relationship between photogenic score and embarrassment score for each face. The photogenic score was measured inside the scanner, and the embarrassment score was measured outside the scanner. Participants experienced strong embarrassment when they viewed their own face images that were evaluated as very bad

allowed the evaluation of modulation of the neuronal activities that co-varied with embarrassment. First, we specified the brain regions that were more strongly activated during self-evaluation than during the evaluation of others. We found significant activations in the right prefrontal cortex (PFC), bilateral insular cortex, bilateral occipital cortex, and anterior cingulate cortex. The significant activations in the PFC were found only in the right hemisphere. The posterior cluster was located in the inferior part of the precentral gyrus, which corresponded to Brodmann's area (BA) 6 or 44. By contrast, the anterior cluster was located in the middle inferior frontal gyrus (mIFG), corresponding to BA 45 or 46 (Fig. 4).

\section{Right mIFG for self-evaluation}

Within the areas activated by the contrast of SELF versus OTHERS, we identified those where the activity in the SELF condition co-varied with the embarrassment scores (Fig. 4). We did not identify any regions that showed a positive correlation with the scores. However, we found a region that showed a negative correlation with the scores in the right PFC. This area was included in the anterior cluster, and so was located in the mIFG. It was selectively activated during the evaluation of self face images, but not during the evaluation of others. The activation in this area was not modulated by the extent of public self-consciousness. This 
Fig. 4 Activation pattern of the right mIFG (light blue). From within the areas identified as active by the contrast of SELF versus OTHERS (a), we identified the brain regions where the activity in the SELF condition co-varied with the embarrassment scores. The right $\mathrm{mIFG}$ showed a negative correlation with the scores (b). The right mIFG was selectively activated during the evaluation of own face images, but not during the evaluation of others (c). The right ventral precentral gyrus (dark blue, d) responded to both self and other faces. The activation showed a significant positive correlation with the public self-consciousness score (blue closed circle, e) of the selfconsciousness scale. There was no significant correlation with the private self-consciousness score (magenta closed triangle)

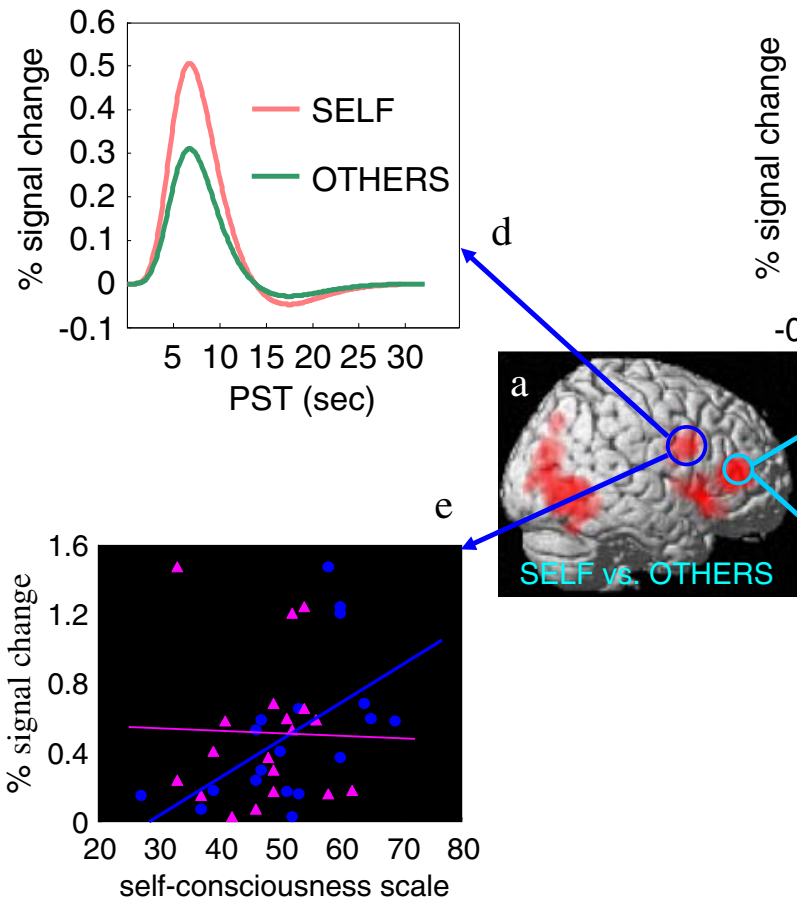

negative correlation made it unlikely that the right mIFG represented the induced embarrassment itself. As the right mIFG was selectively activated during the evaluation of one's own face, but not the faces of others, it might be related to the self-evaluation that induces embarrassment. The right lateral PFC is involved in self-referential processing, including autobiographical memory retrieval (Fink et al. 1996; Vogeley et al. 1999), or the evaluation of traits concerning the self (Ochsner et al. 2005; Schmitz et al. 2004). Schmitz and colleagues suggested that the increased activity in the right PFC during self-evaluation is associated with increased self-relevance. Taken together, these findings suggest that the right mIFG is selectively engaged in self-evaluation, reflecting self-relevance (Schmitz et al. 2004).

\section{Right ventral precentral gyrus}

The right ventral precentral gyrus, which is part of the ventral premotor area, showed activation during face recognition, and was more strongly activated when viewing one's own face, although it was still responsive to others' faces. The "mirror neuron" network including the ventral premotor area has been suggested to be important for imitation (Iacoboni and Mazziotta 2007) in order to establish communication between individuals via a simulation mechanism that maps the actions of others onto one's own motor repertoire (Uddin et al. 2005). Uddin and colleagues postulated the "mirror neuron" systems are activated by self-face recognition because of the ease with which one can map oneself onto one's own motor system (Uddin et al. 2005).

Furthermore, activation of the right ventral precentral gyrus is reportedly modulated by the extent of public selfconsciousness. People with high public self-consciousness tend to react strongly and become publicly self-aware when they are exposed to inducers of public self-awareness, such as perceptual feedback (Buss 1980). As mentioned previously, self images in a mirror can induce a coy reaction or exposure embarrassment that reflects lower-level selfawareness at the age of 24 months. An autism study by Dawson and McKissick showed that self-recognition measured by the mark test and imitation were not directly related, but rather imitation was related to public selfawareness (Dawson and McKissick 1984). These findings suggest the existence of a psychological construct such as "interest in self" (Fig. 5) (Akagi 2003) that is represented in the right ventral precentral gyrus. Interest in self is related to low-level self-awareness that cannot be measured by the mark test, but is manifested by exposure embarrassment (Akagi 2003).

In several pathological conditions, interest in self might be lacking. For instance, autistic children are able to recognize their own image in a mirror, but they display a neutral reaction to such images without demonstrable feelings of exposure 
Development of Self and related neural representation

\begin{tabular}{|l|l|l|l|l|l|}
\hline Age (Mo) & Construct & Related construct & $\begin{array}{l}\text { Related self-conscious } \\
\text { emotion }\end{array}$ & $\begin{array}{l}\text { Corresponding neural } \\
\text { substrates (by adults fMRI) }\end{array}$ & $\begin{array}{l}\text { Self-related activity } \\
\text { (by adults fMRI) }\end{array}$ \\
\hline 18 & Self recognition & & & Right parieto-premotor & \\
\hline 24 & $\begin{array}{l}\text { Self awareness } \\
\text { low level }\end{array}$ & Interest in self & $\begin{array}{l}\text { Exposure-embarrassment } \\
\text { empathy jealousy }\end{array}$ & Right PMv & $\begin{array}{l}\text { Positive correlation with } \\
\text { public self consciousness scale }\end{array}$ \\
\hline $30-36$ & $\begin{array}{l}\text { Self awareness } \\
\text { high level }\end{array}$ & Self evaluation & $\begin{array}{l}\text { Evaluative-embarrassment } \\
\text { guilty pride }\end{array}$ & Right middle IFG & $\begin{array}{l}\text { Negative correlation with } \\
\text { embarrassment }\end{array}$ \\
\hline
\end{tabular}

Fig. 5 Revised model of development of self and self-conscious emotion. $P M v$, ventral premotor cortex; $I F G$, inferior frontal gyrus

embarrassment (Dawson and McKissick 1984; Spiker and Ricks 1984). Abused children also display a neutral or negative reaction to their image as reflected in a mirror, with little evidence of self-conscious emotion (Schneider-Rosen and Cicchetti 1991). Thus, interest in self might be an important element that creates links across imitation, selfawareness, and autism. Future studies are needed to clarify the detailed relationships among these functions.

\section{Implication of fMRI results for the model of development of self}

As self-recognition and self-evaluation functions emerge at different stages of development (Fig. 5), the period of maturation of the two prefrontal regions might differ correspondingly. During the period between 18 months and 2 years when self-recognition is acquired, infants also begin to imitate the intentional actions of other people. The interest in self and the associated exposure embarrassment, and the imitation of the intentional actions of other people, are both accompanied by activation of the ventral premotor region. Thus, the ventral premotor region might well begin to develop and to function at this time. The self-evaluation and recall of autobiographical memories emerge at the same time (around the age of 3 years). Thus, the mIFG, which is related to the processing of higher-level self-related information, matures later than the ventral premotor region.

These results present new possibilities for identifying the order of maturation of the neural representation of specific functions through the timing of their emergence (Fig. 5). The functional neuroimaging of infants is still limited in terms of the measurements and tasks that can be applied (but, see below); however, direct investigation of the brain functions of children is not necessarily the only effective way of studying brain development. As was proposed earlier, combining the past knowledge accumulated in the field of cognitive neuroscience with that from the field of developmental psychology could shed new light on the development of the brain. This type of approach, combining the extraction of landmark behavior, brain localization, and model formation, can be applied to all stages of social cognitive development, and necessi- tates close collaborations between neuroimagers and developmental psychologists.

\section{Direct measures of the infant brain: future perspectives}

In the school age group ( $>6$ years old), it is possible to conduct structural MRI or even functional MRI in awake condition. This is because at this age, the majority of children can follow the instructions to stay still in the closed space in the magnet for about half an hour, and to conduct specific tasks. However, in younger age group ( $<6$ years old), it is almost impossible.

The development of the technology needed to apply neuroimaging techniques to pediatric groups is ongoing. Near-infrared spectroscopy (NIRS) is one of the most promising approaches for use in infants (Aslin and Mehler 2005). NIRS is a non-invasive neuroimaging technique that measures the change in oxy-/deoxy-hemoglobin concentration in the cerebral blood vessels that reflects neuronal activity. As NIRS utilizes near-infrared light, it is highly applicable to infants in naturalistic conditions. Recent advances in NIRS have facilitated the accurate and noninvasive measurement of time-course changes of the oxyhemoglobin concentration $([\mathrm{HbO}])$ and deoxyhemoglobin concentration ([HbR]) during neural activation in humans (for a review, see Gibson et al. 2005; Villringer et al. 1993, 1994). Simultaneous measurements of NIRS and fMRI data have indicated that these two methodologies are well correlated (Kleinschmidt et al. 1996; Punwani et al. 1998; Toyoda et al. 2008). Since the mid-1990s, NIRS has been used in awake human infants in conjunction with tasks that assess visual (Taga et al. 2003), memory, and language functions. Recently, NIRS has been applied to infants in conjunction with sophisticated tasks that are relevant to aspects of developmental social psychology, such as face preferences (Nakato et al. 2007; Otsuka et al. 2007), and language-specific phonemic discrimination (MinagawaKawai et al. 2007). Thus, despite some technical challenges (such as those related to the attachment of probes, limitations of the spatial resolution and coverage field, and co-registration with anatomical data), in concert with electrophysiological techniques (such as electroencephalog- 
raphy and event-related potential), NIRS is "emerging as a third noninvasive window into the infant brain" (Aslin and Mehler 2005) (Fig. 1).

Acknowledgments This study was supported, in part, by a Grant-inAid for Scientific Research (S\#17100003 to N.S.) from the Japan Society for the Promotion of Science.

Open Access This article is distributed under the terms of the Creative Commons Attribution Noncommercial License which permits any noncommercial use, distribution, and reproduction in any medium, provided the original author(s) and source are credited.

\section{References}

Akagi, K. (2003). Review of the research on mirror self-recognition. Perspectives from developmental psychopathology. Human Sciences Research, 11, 51-62. in Japanese.

Alessandri, S. M., \& Lewis, M. (1993). Parental evaluation and its relation to shame and pride in young children. Sex Roles, 29, 335-343. doi:10.1007/BF00289427.

Amsterdam, B. (1972). Mirror self-image reactions before age two. Developmental Psychobiology, 5, 297-305. doi:10.1002/ dev.420050403.

Aslin, R. N., \& Mehler, J. (2005). Near-infrared spectroscopy for functional studies of brain activity in human infants: promise, prospects, and challenges. Journal of Biomedical Optics, 10, 1-3. doi: $10.1117 / 1.1854672$.

Baron-Cohen, S., Tager-Flusberg, H., \& Cohen, D. J. (1993). Understanding other minds: perspectives from autism, Oxford University Press.

Bischof-Köhler, D. (1991). The development of empathy in infants. In M. E. Lamb, \& H. Keller (Eds.), Infant development: Perspectives from German-speaking countries. Hillsdale, NJ: Erlbaum.

Brothers, L. (1990). The social brain: a project for integrating primate behavior and neurophysiology in a new domain. Concepts in Neuroscience, 1, 27-151.

Buss, A. H. (1980). Self-consciousness and social anxiety. San Francisco: Freeman.

Dapretto, M., Davies, M. S., Pfeifer, J. H., Scott, A. A., Sigman, M., Bookheimer, S. Y., et al. (2006). Understanding emotions in others: mirror neuron dysfunction in children with autism spectrum disorders. Nature Neuroscience, 9, 28-30. doi:10.1038/nn1611.

Dawson, G., \& McKissick, F. C. (1984). Self-recognition in autistic children. Journal of Autism and Developmental Disorders, 14, 383-394. doi:10.1007/BF02409829.

Duval, S., \& Wicklund, R. A. (1972). A theory of objective selfawareness. New York: Academic.

Fink, G. R., Markowitsch, H. J., Reinkemeier, M., Bruckbauer, T., Kessler, J., \& Heiss, W. D. (1996). Cerebral representation of one's own past: neural networks involved in autobiographical memory. The Journal of Neuroscience, 16, 4275-4282.

Gallup Jr., G. G. (1970). Chimpanzees: self-recognition. Science, 167, 86-87. doi:10.1126/science.167.3914.86.

Gibson, A. P., Hebden, J. C., \& Arridge, S. R. (2005). Recent advances in diffuse optical imaging. Physics in Medicine and Biology, 50, R1-R43. doi:10.1088/0031-9155/50/4/R01.

Iacoboni, M., \& Dapretto, M. (2006). The mirror neuron system and the consequences of its dysfunction. Nature Reviews. Neuroscience, 7, 942-951. doi:10.1038/nrn2024.
Iacoboni, M., \& Mazziotta, J. C. (2007). Mirror neuron system: basic findings and clinical applications. Annals of Neurology, 62, 213218. doi:10.1002/ana.21198.

Kleinschmidt, A., Obrig, H., Requardt, M., Merboldt, K.-D., Dirnagl, U., Villinger, A., et al. (1996). Simultaneous recording of cerebral blood oxygenation changes during human brain activation by magnetic resonance imaging and near-infrared spectroscopy. Journal of Cerebral Blood Flow and Metabolism, 16, 817-826. doi:10. 1097/00004647-199609000-00006.

Lewis, M. (1997). The self in self-conscious emotions. Annals of the New York Academy of Sciences, 818, 119-142. doi:10.1111/j. 1749-6632.1997.tb48251.x.

Lewis, M., Sullivan, M. W., Stranger, C., \& Weiss, M. (1989). Self development and self-conscious emotions. Child Development, 60, 146-156. doi:10.2307/1131080.

Minagawa-Kawai, Y., Mori, K., Naoi, N., \& Kojima, S. (2007). Neural attunement processes in infants during the acquisition of a language-specific phonemic contrast. The Journal of Neuroscience, 27, 315-321. doi:10.1523/JNEUROSCI.198406.2007.

Morin, A. (2006). Levels of consciousness and self-awareness: a comparison and integration of various neurocognitive views. Consciousness and Cognition, 15, 358-371. doi:10.1016/j.concog. 2005.09.006.

Morita, T., Itakura, S., Saito, D. N., Nakashita, S., Harada, T., Kochiyama, T., et al. (2008). The role of the right prefrontal cortex in self-evaluation of the face: a functional magnetic resonance imaging study. Journal of Cognitive Neuroscience, 20, 1-14. doi:10.1162/jocn.2008.20024.

Nakato, E., Otsuka, Y., Kanazawa, S., Yamaguchi, M. K., Watanabe, S., \& Kakigi, R. (2007). When do infants differentiate profile face from frontal face? a near-infrared spectroscopic study. Human Brain Mapping, in press.

Ochsner, K. N., Beer, J. S., Robertson, E. R., Cooper, J. C., Gabrieli, J. D., Kihsltrom, J. F., et al. (2005). The neural correlates of direct and reflected self-knowledge. NeuroImage, 28, 797-814. doi:10.1016/j. neuroimage.2005.06.069.

Otsuka, Y., Nakato, E., Kanazawa, S., Yamaguchi, M. K., Watanabe, S., \& Kakigi, R. (2007). Neural activation to upright and inverted faces in infants measured by near infrared spectroscopy. NeuroImage, 34, 399-406. doi:10.1016/j.neuroimage.2006.08.013.

Platek, S. M., Keenan, J. P., Gallup, G. G. J., \& Mohamed, F. B. (2004). Where am I? the neurological correlates of self and other. Brain Research. Cognitive Brain Research, 19, 114-122. doi:10.1016/j.cogbrainres.2003.11.014.

Punwani, S., Ordidge, R. J., Cooper, C. E., Amess, P., \& Clemence, M. (1998). MRI measurements of cerebral deoxyghemoglobin concentration $[\mathrm{dHb}]$ - correlation with near infrared spectroscopy (NIRS). NMR in Biomedicine, 11, 281-289. doi:10.1002/(SICI) 1099-1492(199810)11:6<281::AID-NBM529>3.0.CO;2-6.

Schaffer, H. R. (1996). Social development: An introduction. London: Blackwell.

Schmitz, T. W., Kawahara-Baccus, T. N., \& Johnson, S. C. (2004). Metacognitive evaluation, self-relevance, and the right prefrontal cortex. NeuroImage, 22, 941-947. doi:10.1016/j.neuroimage.2004.02.018.

Schneider-Rosen, K., \& Cicchetti, D. (1991). Early self-knowledge and emotional development: visual self-recognition and affective reactions to mirror self-images in maltreated and non-maltreated toddlers. Developmental Psychology, 27, 471-478. doi:10.1037/ 0012-1649.27.3.471.

Spiker, D., \& Ricks, M. (1984). Visual self-recognition in autistic children: developmental relationships. Child Development, 55, 214-225. doi:10.2307/1129846.

Sugiura, M., Sassa, Y., Jeong, H., Miura, N., Akitsuki, Y., Horie, K., et al. (2006). Multiple brain networks for visual self-recognition 
with different sensitivity for motion and body part. NeuroImage, 32, 1905-1917. doi:10.1016/j.neuroimage.2006.05.026.

Sugiura, M., Watanabe, J., Maeda, Y., Matsue, Y., Fukuda, H., \& Kawashima, R. (2005). Cortical mechanisms of visual selfrecognition. NeuroImage, 24, 143-149. doi:10.1016/j.neuroimage. 2004.07.063.

Sullivan, M. W., Bennett, D. S., \& Lewis, M. (2003). Darwin's view Selfevaluative emotions as context-specific emotions. Annals of the New York Academy of Sciences, 1000, 304-308. doi:10.1196/ annals.1280.031.

Taga, G., Asakawa, K., Maki, A., Konishi, Y., \& Koizumi, H. (2003). Brain imaging in awake infants by near-infrared optical topography. Proceedings of the National Academy of Sciences of the United States of America, 100, 10722-10727. doi:10.1073/ pnas. 1932552100.

Toyoda, H., Kashikura, K., Okada, T., Nakashita, S., Honda, M., Yonekura, Y., et al. (2008). Source of nonlinearity of the BOLD response revealed by simultaneous fMRI and NIRS. NeuroImage, 39, 997-1013. doi:10.1016/j.neuroimage.2007.09.053.

Uddin, L. Q., Kaplan, J. T., Molnar-Szakacs, I., Zaidel, E., \& Iacoboni, M. (2005). Self-face recognition activates a frontoparietal "mirror" network in the right hemisphere: an event-related fMRI study. NeuroImage, 25, 926-935. doi:10.1016/j.neuroimage.2004.12.018.

Villringer, A., Planck, J., Hock, C., Schleinkofer, L., \& Dirnagl, U. (1993). Near infrared spectroscopy (NIRS): a new tool to study hemodynamic changes during activation of brain function in human adults. Neuroscience Letters, 154, 101-104. doi:10.1016/ 0304-3940(93)90181-J.

Villringer, A., Planck, J., Stodieck, S., Botzel, K., Schleinkofer, L., \& Dirnagl, U. (1994). Noninvasive assessment of cerebral hemodynamics and tissue oxygenation during activation of brain cell function in human adults using near infrared spectroscopy. Advances in Experimental Medicine and Biology, $345,559-565$.

Vogeley, K., Kurthen, M., Falkai, P., \& Maier, W. (1999). Essential functions of the human self model are implemented in the prefrontal cortex. Consciousness and Cognition, 8, 343-363. doi:10.1006/ccog.1999.0394.

Willingham, D. T., \& Dunn, E. W. (2003). What neuroimaging and brain localization can do, cannot do, and should not do for social psychology. Journal of Personality and Social Psychology, 85, 662-671. doi:10.1037/0022-3514.85.4.662. 